\title{
The role of VEGF in psoriasis: an update
}

\author{
Agnieszka Gerkowicz', Mateusz Socha ${ }^{2}$, Aldona Pietrzak', \\ Tomasz Zubilewicz ${ }^{3}$, Dorota Krasowska' \\ 'Department of Dermatology, Venereology and Paediatric Dermatology, Medical University of Lublin, Poland \\ ${ }^{2}$ Department of Internal Medicine and Cardiology, First Military Clinical Hospital with the Outpatient Clinic, Lublin, Poland \\ ${ }^{3}$ Chair and Department of Vascular Surgery and Angiology, Medical University of Lublin, Poland
}

\begin{abstract}
Psoriasis is a common, chronic immune-mediated multifactorial skin disease. In its pathogenesis altered differentiation and hyperproliferation of keratinocytes, dysregulation of immunological cell functions, together with abnormal angiogenesis are involved. Angiogenesis is defined as the formation of new blood vessels from the pre-existing vascular bed. This complex and multistep process is regulated by different factors among which vascular endothelial growth factor (VEGF) is considered to be the most important. The aim of this paper is a review of the current literature considering the role of vascular endothelial growth factor in psoriasis. Many studies have focused on the role of VEGF in psoriasis and revealed its increased serum and tissue levels which correlated with disease severity. Recent data indicate that VEGF is not only responsible for angiogenesis, but also regulates keratinocyte differentiation. Moreover, it has been suggested that vascular endothelial growth factor could be a link between psoriasis and its comorbidities. So far, there are single clinical cases that reported clearance of psoriasis after anti-VEGF therapy. Therefore, the VEGF pathway might be a potential new therapeutic alternative leading to improvement of psoriasis. However, further clinical studies are needed to evaluate the efficacy and safety of this therapy in psoriasis.
\end{abstract}

Key words: psoriasis, angiogenesis, VEGF

Acta Angiol 2018; 24, 4: 134-140

\section{Introduction}

Psoriasis is a chronic immune-mediated multifactorial skin disease affecting $\mathrm{I}-3 \%$ of the world's population $[1,2]$. Recent studies have improved our understanding of the immunopathological mechanisms of psoriasis and therefore facilitated its therapy [3]. Apart from dysregulation of immunological cell functions, the altered differentiation and hyperproliferation of keratinocytes together with abnormal angiogenesis are involved in the development of psoriasis [I, 2].

In psoriasis, the first pathological change is vascular proliferation, with the dermal papilla blood vessels being dilated, elongated and tortuous [2, 4]. Lympho- cytic infiltrate and oedema are also present [4]. These changes appear before epidermal hyperplasia becomes apparent. Psoriatic plaques clear after these changes disappear $[5,6]$. Clinically, vascular proliferation manifests as the Auspitz's sign [7]. Apart from the morphological changes described, there are also ultrastructural transformations in the capillaries. As shown with the use of electron microscopy, features typical of venous capillaries, e.g. basement membrane (single or multi-layered) or endothelial bridged fenestration, are displayed by capillary loops in psoriatic plaques, unlike healthy skin capillaries, which preserve features characteristic of arterial vessels $[5,6]$. Treatment results in the remodelling of the former into arterial type capillaries [6]. 
Since angiogenesis defined as the formation of new blood vessels from the pre-existing vascular bed is a crucial component of psoriasis pathogenesis, it is of special interest to identify factors responsible for angiogenesis control. Vascular endothelial growth factor (VEGF) is considered the main factor responsible for regulating angiogenesis. The VEGF superfamily includes VEGF-A, VEGF-B, VEGF-C, VEGF-D, VEGF-E and placental growth factor (PLGF). However, the term VEGF usually refers to VEGF-A, which is the main member of the VEGF superfamily [8,9]. Different cell types can express VEGF, including monocytes/ macrophages, keratinocytes, fibroblasts, osteoblasts, astrocytes and tumour cells [10-12]. The main inducer of VEGF synthesis is low oxygen concentration [5]. An important role in the induction of VEGF expression is also played by different proinflammatory cytokines: interleukins 8 and I7A (IL-8, IL-I7A), tumour necrosis factor alpha (TNF- $\alpha$ ), some growth factors: basic fibroblast growth factor (bFGF), transforming growth factor-beta (TGF- $\beta$ ), and prostaglandins. Hypoglycaemia also increases its synthesis [5, I3-I5].

VEGF exerts its biological function through its receptors. There are three VEGF receptors belonging to the kinase family: vascular endothelial growth factor receptor-I(VEGFR-I), vascular endothelial growth factor receptor-2 (VEGFR-2) also called kinase insert domain-containing receptor (KDR) and vascular endothelial growth factor receptor-3 (VEGFR-3). The first two types are expressed mainly by blood vascular endothelium; however, they were also identified on various cell types, including monocytes, macrophages, haematopoietic stem cells, platelets or vascular smooth muscle cells [16, 17], whereas VEGFR-3 expression is restricted to the lymphatic endothelium [17]. Apart from the receptors described above, VEGF can also bind to neuropilin-I (NRP-I) and neuropilin-2 (NRP-2). It was demonstrated that NRP-I binds VEGF-A and acts as co-receptor for VEGFR-2, which leads to enhancement of VEGF signalling and function [18]. Herzog et al. [18] found that VEGF binding to NRP-I has specific effects on endothelial cell signalling and is important for endothelial cell migration and angiogenesis mediated via complex formation between NRP-I and KDR. It also increases signalling to focal adhesions, whereas NRP-2 together with VEGFR-3 is involved in mediating VEGF-C signalling in lymphatic vessels [19].

VEGF exerts the most potent effect on endothelial cells by stimulating their proliferation, migration and inhibiting endothelial cell apoptosis. Moreover, it increases vascular permeability and stimulates blood vessel dilation [20]. VEGF also acts as a chemoattractant for leukocytes, stimulates matrix metalloproteinase (MMP) synthesis and expression of adhesive mole- cules, and regulates the function of dendritic cells and lymphocytes. Due to its multidirectional action, VEGF may be involved in different inflammatory processes, autoaggressive reactions or allergic reactions [2I].

The aim of this paper is a review of the current literature considering the role of VEGF in psoriasis.

\section{VEGF in psoriasis}

Many studies have focused on the role of VEGF in psoriasis. It was demonstrated that VEGF transgenic mice had an increased density of tortuous cutaneous blood capillaries accompanied by elevated expression of VEGFR-I and VEGFR-2 [22]. Then Xia et al. [23] observed that an excess of VEGF in KI4-VEGF transgenic mice can promote the development of a phenotype resembling human psoriasis, including elongated and enlarged vessels in the dermal papillae, abnormal epidermal proliferation and differentiation with parakeratosis and hyperkeratosis found in skin biopsies. Interestingly, the psoriasis-like lesions occurred in mice older than 5 months, which suggests that chronic overexpression of VEGF is required for the development of these lesions. Moreover, the authors demonstrated that the psoriasis-like phenotype can be normalized after VEGF blockade [23]. Following these observations, many studies were conducted to evaluate the role of human cutaneous VEGF in psoriasis. VEGF can be expressed by keratinocytes; however, the level of this growth factor is low in normal epidermis and increases in skin diseases associated with angiogenesis and chronic inflammation [24]. Sankar et al. [6] observed that VEGF expression levels in skin biopsies taken from psoriatic plaques were significantly higher compared to the healthy controls. Similar results were reported by other authors $[25,26]$. Data considering the correlation between VEGF levels in skin biopsies and psoriasis area and severity index (PASI) are inconclusive. Some studies have demonstrated a significant correlation [6], whereas other studies reported none [27]. Simonetti et al. [25] observed that VEGF overexpression in psoriatic skin correlated with hypoxia-inducible factor-I alpha (HIF-I $\alpha$ ) and MMP-2 expression, which indicates that VEGF could play an important role in the link between inflammation and angiogenesis. Rashed et al. [26] found increased VEGF expression in the epithelium and moderate expression within inflammatory infiltrate and vessels. They also reported a significant positive correlation between VEGF, inducible nitric oxide synthase (iNOS) and survivin. Like VEGF, survivin and iNOS were expressed at significantly higher levels in psoriatic plaques in comparison with healthy controls. This confirms that VEGF promotes endothelial cell survival and new blood vessel formation, and thus may play an important role in the development 
of psoriasis. Contrary to those studies, Liew at al. [27] found no significant correlation between VEGF-A levels in skin biopsies taken from patients with psoriasis and healthy volunteers. Nevertheless, the authors observed that in psoriatic skin, the intensity of staining for VEGF-C was significantly higher in comparison to healthy controls. Moreover in patients with psoriasis nerve growth factor (NGF) was significantly expressed compared with healthy controls. According to the authors, this might suggest a synergistic interplay between NGF and VEGF-C in psoriasis, and this interplay could be a potential new therapeutic target in psoriasis vulgaris [27]. Therefore the role of VEGF-C in psoriasis was studied. Moustou et al. observed higher expression of VEGF-C and VEGF-D on lymphatic vessels within psoriatic plaques than in the non-psoriatic adjacent skin. While etanercept therapy decreased expression of VEGF-C and VEGF-D. These findings suggest the presence of lymphatic vessels remodelling in psoriasis. However, further studies are necessary to clarify its role in the development and recession of psoriasis [28].

As mentioned above, VEGF exerts its biological action through its receptors. Man et al. [29] demonstrated the presence of VEGF receptors on keratinocytes in normal human epidermis. Later, the authors found that in normal healthy skin, VEGFR-I and VEGFR-2 were expressed only in the basal and suprabasal layer, whereas in patients with psoriasis those receptors were expressed in keratinocytes in all layers of the epidermis within psoriatic plaques and in all layers except the stratum corneum in perilesional and non-lesional keratinocytes. Analysis of VEGFR mRNA and protein levels of VEGFRs revealed the highest levels in lesional keratinocytes in comparison to perilesional, non-lesional or healthy skin. Considering the presence of VEGFRs in parakeratotic stratum corneum within psoriatic plaques, it was suggested that expression of those receptors may correlate with keratinocyte differentiation [30]. Then in a recent study, it was demonstrated that overexpression of VEGFR-2 in psoriatic epidermis is associated with upregulation of keratins $(\mathrm{K})$ : K6, KI6, KI7, typical for incomplete keratinocyte differentiation, and downregulation of $\mathrm{KI}$ and $\mathrm{KIO}$, which are characteristic for complete terminal differentiated keratinocytes [3I]. Later the authors found that VEGF upregulates the expression of $\mathrm{K} 6, \mathrm{KI} 6$ and $\mathrm{KI} 7$ in normal human epidermal keratinocytes via the STAT3 (signal transducer and activator of transcription), ERKI/2 (extracellular regulated protein kinase I/2) and p38 pathways, whereas the VEGF-induced decrease of $\mathrm{KI}$ and $\mathrm{KIO}$ expression in normal human epidermal keratinocytes is due to activation of the ERKI/2 pathway. This study casts a new light on the role of VEGF in psoriasis and demonstrates that VEGF may promote aberrant keratin expression resulting in the keratinocyte hyperproliferation typical for psoriasis [3I].

Apart from local overexpression of VEGF, it was demonstrated that patients with psoriasis have increased serum levels of VEGF and these levels correlated with PASI score [I, 32-34]. Moreover, serum VEGF concentrations correlated with psoriasis duration [34]. Flisiak et al. [I] observed that elevated serum concentrations of VEGF decreased after topical therapy but remained higher than in healthy controls. Interestingly, the concentration of soluble vascular endothelial growth factor receptor-I (sVEGFR-I) remained significantly higher than in a control group regardless of treatment. According to the authors, it cannot be excluded that elevated levels of sVEGFR-I are required to bind VEGF and prevent further proangiogenic signal transduction [I]. Andrys et al. [35] reported a significant reduction of plasma VEGF levels after combined therapy with UVB and coal tar [35]. Contrary to those studies, Akman et al. [36] found elevated plasma levels of VEGF in psoriatic patients in comparison to healthy controls, but the differences were not statistically significant. Moreover, VEGF levels decreased after PUVA therapy but increased after narrow band UVB (NB-UVB) and Re-PUVA. It cannot be excluded that changes in VEGF levels may result from increased epidermal proliferation after UVB exposure and individual response to systemic retinoids due to the rebound phenomenon [37]. However, further studies are required to explain these findings. In another study, the association between serum VEGF levels and psoriasis severity was not confirmed [38]. Yamamoto et al. [39] reported elevated serum levels of VEGF in a patient with severe generalised pustular psoriasis accompanied by blister formation and its decrease with remission. According to the authors, observed blister formation resulted from systemic administration of steroids that exacerbated psoriasis and enhanced microvascular hyperpermeability. It has been suggested that increased circulating plasma levels of VEGF in patients with severe psoriasis can induce hyperpermeability of renal vessels, leading to proteinuria [40] Increased VEGF levels were also observed in psoriatic arthritis and correlated with CRP and disease duration [4I].

Genetic studies have provided novel data regarding the role of VEGF in psoriasis. The VEGF gene is located on 6p2I.3 near the PSORSI chromosomal locus for psoriasis susceptibility [7, 42]. Further studies demonstrated that two groups of psoriatic patients can be distinguished on the basis of their VEGF genotype: patients with "low and high VEGF production" [43]. Patients with high VEGF production are characterized by early psoriasis onset and a severe disease course [44]. Therefore, it has been suggested that individuals 
with a high VEGF production may develop a "proangiogenic" psoriasis phenotype, which has therapeutic implications [7].

Since more than 250 aberrantly expressed MircroRNAs (miRNAs) in psoriasis were identified, many studies focused on its role in this disease $[45,46]$. A possible link between miRNAs and VEGF expression was also studied [46-48]. Li et al. [46] observed that expression of HIF- $I \alpha$ and VEGF-A were down-regulated by miR-I 50 overexpression while promoted by miR-I50 inhibition in $\mathrm{HaCaT}$ cells and primary adult human keratinocytes (HKCs). Xu et al. [49] suggested that miR203 expression may be upregulated by IL- 17 stimulation, and miR203 is a positive regulator of IL-17-induced VEGF secretion. Evaluation of the role of specific miRNAs in psoriasis and regulation of angiogenesis seems to be important, but more studies are necessary.

It is well known that psoriasis is associated with many comorbidities, including diabetes, lipid abnormalities, obesity and metabolic syndrome. Patients with psoriasis have an increased risk of cardiovascular diseases such as atherosclerosis [7]. Significantly, angiogenesis plays an important role in the development of both psoriasis and atherosclerosis. In psoriasis, local levels of VEGF in psoriatic plaques are elevated and correlate with disease severity; the same is true for VEGF levels within the atherosclerotic plaque. Likewise, the "high VEGF-producing" genotype $(+405 \mathrm{CC})$ in psoriasis this genotype is associated with a poor prognosis in patients with chronic heart failure [7]. In both diseases, at the beginning, local hypoxia triggers the release of hypoxia-inducible factor I (HIF-I) with a further expression of VEGF which leads to the formation of new vessels and promotes lymphocyte migration via increased expression of adhesive molecules [7, 22]. Moreover, oxidized phospholipids known as crucial factors in the development of atherosclerotic plaques can enhance VEGF expression in keratinocytes, which suggests their potential role in psoriasis [7]. These data emphasize that VEGF is not only involved in angiogenesis and keratinocyte differentiation but could also be a link between psoriasis and its comorbidities. It has been suggested that enhanced expression of VEGF secondary to one of those diseases may influence or exacerbate the other [7]. Therefore, VEGF or its receptors could be a potent target for future therapies.

\section{VEGF-targeted therapy in psoriasis}

VEGF inhibitors are currently licensed for the treatment of eye diseases or malignancies. Most of them target the VEGF pathway either by direct inhibition of VEGF protein, indirectly by inhibiting tyrosine kinase signalling and by blocking the VEGF receptor, or by preventing the binding of VEGF to its receptor [37, 49]. So far there are single clinical cases that reported clearance of psoriasis after anti-VEGF therapy. Akman et al. [50] reported complete remission of psoriasis after treatment with a monoclonal antibody against VEGF (bevacizumab) in a 60-year old man with metastatic colon cancer and long-lasting severe psoriasis. Later, Datta-Mitra et al. [5I] reported complete remission of both psoriasis and psoriatic arthritis in a 65-year old male patient with metastatic renal cell carcinoma who received bevacizumab. Importantly, at the beginning, the patient received combined therapy with bevacizumab and interferon-alpha, which led to significant improvement of both psoriasis and psoriatic arthritis after 3 months. However, due to development of proteinuria, he was switched to treatment with tyrosine kinase inhibitors, during which his serum creatinine levels increased and a relapse of both psoriasis and psoriatic arthritis was observed. After re-initiation of monotherapy with bevacizumab, tumour stabilization, as well as, remission of psoriasis and psoriatic arthritis was achieved [5I]. Contrary to the previous study, other authors observed remission of psoriasis during treatment with tyrosine kinase inhibitors [52, 53]. Recently, it has been demonstrated in an imiquimodinduced mouse model of psoriasis that topical sunitinib can reduce imiquimod-induced psoriasis-like inflammation [54]. As mentioned above, targeting VEGF signalling might be a promising therapeutic option. However, it cannot be excluded that an additional factor might also have a beneficial effect on psoriasis during this oncologic therapy [37]. One of the most important triggering factors in psoriasis is stress. Therefore, stabilisation of cancer during anti-VEGF treatment may reduce stress and improve the course of psoriasis [37]. A further aspect that has to be considered during anti-VEGF therapy in psoriatic patients are side effects such as hypertension, left ventricular dysfunction or gastrointestinal perforation. Cardiovascular complications are of special interest since psoriatic patients have increased cardiovascular risk [37]. Apart from novel experimental therapies, there is evidence that traditional medications also affect angiogenesis. It was found that inhibiting the activity of transcription factors of the nuclear factor of activated $T$ cells family by cyclosporine $A$ decreases migration of primary endothelial cells and VEGF-induced angiogenesis [49]. Therapy with TNF inhibitors also reduces VEGF expression, leading to a decreased blood supply [49].

To sum up, a growing body of evidence suggests an important role of VEGF and its receptors in psoriasis. Recent data indicate that VEGF is not only responsible for angiogenesis, but also regulates keratinocyte differentiation. The results of many studies indicate 
that targeting the VEGF pathway might be a potential new therapeutic alternative leading to improvement of psoriasis. However, further clinical studies are needed to evaluate the efficacy and safety of this therapy in psoriasis.

\section{Conflict of interest}

None.

\section{Funding source}

The study was supported by the Medical University of Lublin funds (grant numbers: DS 168/20I7).

\section{References:}

I. Flisiak I, Zaniewski P, Rogalska M, et al. Effect of psoriasis activity on VEGF and its soluble receptors concentrations in serum and plaque scales. Cytokine. 2010; 52(3): 225-229, doi: 10.1016/j. cyto.2010.09.012, indexed in Pubmed: 20980160.

2. Wang $X$, Sun $X, Q u$, et al. Overexpressed fibulin-3 contributes to the pathogenesis of psoriasis by promoting angiogenesis. Clin Exp Dermatol. 2018 [Epub ahead of print], doi: 10.1 III/ ced. 13720 , indexed in Pubmed: 3014675I.

3. Ogawa E, Sato Y, Minagawa A, et al. Pathogenesis of psoriasis and development of treatment. J Dermatol. 2018; 45(3): 264-272, doi: 10.1111/1346-8138.14139, indexed in Pubmed: 29226422.

4. De Rosa G, Mignogna C. The histopathology of psoriasis. Reumatismo. 2007; 59 Suppl I: 46-48, indexed in Pubmed: 17828343.

5. Heidenreich R, Röcken M, Ghoreschi K. Angiogenesis drives psoriasis pathogenesis. Int J Exp Pathol. 2009; 90(3): 232-248, doi: $10.1111 /$ /j. 1365-2613.2009.00669.x, indexed in Pubmed: 19563608.

6. Sankar L, Arumugam D, Boj S, et al. Expression of angiogenic factors in psoriasis vulgaris. J Clin Diagn Res. 2017; II(3): EC23-EC27, doi: 10.7860/JCDR/2017/23039.9525, indexed in Pubmed: 285 II 390.

7. Malecic N, Young HS. Excessive angiogenesis associated with psoriasis as a cause for cardiovascular ischaemia. Exp Dermatol. 2017; 26(4): 299-304, doi: $10.1111 /$ exd.13310, indexed in Pubmed: 28156019.

8. Ferrara N, Gerber HP, LeCouter J. The biology of VEGF and its receptors. Nat Med. 2003; 9(6): 669-676, doi: 10.1038/ nm0603-669, indexed in Pubmed: $12778 \mid 65$.

9. Holmes K, Roberts OLI, Thomas AM, et al. Vascular endothelial growth factor receptor-2: structure, function, intracellular signalling and therapeutic inhibition. Cell Signal. 2007; 19(10): 2003-2012, doi: 10.1016/j.cellsig.2007.05.013, indexed in Pubmed: 17658244.

10. Detmar M. The role of VEGF and thrombospondins in skin angiogenesis. J Dermatol Sci. 2000; 24 Suppl I: S78-S84, indexed in Pubmed: II I 37400.

II. Namiecińska M, Marciniak K, Nowak JZ. [VEGF as an angiogenic, neurotrophic, and neuroprotective factor]. Postepy Hig
Med Dosw (Online). 2005; 59: 573-583, indexed in Pubmed: 16407796.

12. Skóra J, Biegus J, Pupka A, et al. Molecular basics of angiogenesis. Postepy Hig Med Dosw (Online). 2006; 60: 410-415, indexed in Pubmed: 16921341.

13. Honorati MC, Cattini L, Facchini A. IL-I7, IL-I beta and TNF-alpha stimulate VEGF production by dedifferentiated chondrocytes. Osteoarthritis Cartilage. 2004; 12(9): 683-691, doi: 10.1016/j.joca.2004.05.009, indexed in Pubmed: 15325633.

14. Hori R, Nakagawa T, Yamamoto N, et al. Role of prostaglandin E receptor subtypes EP2 and EP4 in autocrine and paracrine functions of vascular endothelial growth factor in the inner ear BMC Neurosci. 2010; II: 35, doi: 10.1 I86/I47I-2202-II-35, indexed in Pubmed: 20219142.

15. Yamazaki Y, Morita T. Molecular and functional diversity of vascular endothelial growth factors. Mol Divers. 2006; 10(4): 515-527, doi: 10.1007/s I 1030-006-9027-3, indexed in Pubmed: 16972015 .

16. Marina ME, Roman II, Constantin AM, et al. VEGF involvement in psoriasis. Clujul Med. 20I5; 88(3): 247-252, doi: 10.15386/ cjmed-494, indexed in Pubmed: 26609252.

17. Veikkola T, Karkkainen M, Claesson-Welsh L, et al. Regulation of angiogenesis via vascular endothelial growth factor receptors. Cancer Res. 2000; 60(2): 203-212, indexed in Pubmed: 10667560

18. Herzog B, Pellet-Many C, Britton G, et al. VEGF binding to NRPI is essential for VEGF stimulation of endothelial cell migration, complex formation between NRPI and VEGFR2, and signaling via FAK Tyr407 phosphorylation. Mol Biol Cell. 201 ; 22(15): 2766-2776, doi: 10.1091/mbc.E09-12-1061, indexed in Pubmed: 21653826.

19. Parker MW, Linkugel AD, Goel HL, et al. Structural basis for VEGF-C binding to neuropilin- 2 and sequestration by a soluble splice form. Structure. 2015; 23(4): 677-687, doi: 10.1016/j. str.2015.01.018, indexed in Pubmed: 25752543.

20. Ng YS, Krilleke D, Shima DT. VEGF function in vascular pathogenesis. Exp Cell Res. 2006; 312(5): 527-537, doi: 10.1016/j. yexcr.2005.11.008, indexed in Pubmed: 16330026.

21. Koczy-Baron E, Kasperska-Zając A. [The role of vascular endothelial growth factor in inflammatory processes]. Postepy Hig Med Dosw (Online). 2014; 68: 57-65, doi: 10.5604/17322693. 1086360, indexed in Pubmed: 24491896.

22. Detmar M, Brown LF, Schön MP, et al. Increased microvascular density and enhanced leukocyte rolling and adhesion in the skin of VEGF transgenic mice. J Invest Dermatol. 1998; III(I): I-6, doi: 10.1046/j.1523-1747.1998.00262.x, indexed in Pubmed: 9665379.

23. Xia YP, Li B, Hylton D, et al. Transgenic delivery of VEGF to mouse skin leads to an inflammatory condition resembling human psoriasis. Blood. 2003; 102(I): 161-168, doi: 10.1182/ blood-2002-12-3793, indexed in Pubmed: 12649136.

24. Bae ON, Noh M, Chun Y], et al. Keratinocytic Vascular Endothelial Growth Factor as a Novel Biomarker for Pathological Skin Condition. Biomolecules \& Therapeutics. 2015; 23(1): 12-18, doi: 10.4062/biomolther.2014.102.

25. Simonetti O, Lucarini G, Goteri G, et al. VEGF is likely a key factor in the link between inflammation and angiogenesis in psoriasis: results of an immunohistochemical study. Int 
J Immunopathol Pharmacol. 2006; 19(4): 75I-760, doi: 10.1 177/03946320060 1900405, indexed in Pubmed: 17166397.

26. Rashed H, El-Bary EA. Immunohistochemical evaluation of VEGF, survivin, bcl-2 protein and iNOS in the pathogenesis of psoriasis. Egyptian Journal of Pathology. 2012; 32(I): 91-98, doi: 10.1097/0I.xej.0000417556.36570.93.

27. Liew SC, Das-Gupta E, Chakravarthi S, et al. Differential expression of the angiogenesis growth factors in psoriasis vulgaris. BMC Res Notes. 2012; 5: 20I, doi: I0.I I86/I756-0500-5-20I, indexed in Pubmed: 22537619.

28. Moustou AE, Alexandrou P, Stratigos AJ, et al. Expression of lymphatic markers and lymphatic growth factors in psoriasis before and after anti-TNF treatment. An Bras Dermatol. 2014; 89(6): 89I-897, indexed in Pubmed: 25387493.

29. Man XY, Yang XH, Cai SQ, et al. Immunolocalization and expression of vascular endothelial growth factor receptors (VEGFRs) and neuropilins (NRPs) on keratinocytes in human epidermis. Mol Med. 2006; 12(7-8): 127-136, doi: 10.2119/2006-00024. Man, indexed in Pubmed: 17088944.

30. Man XY, Yang $X H$, Cai SQ, et al. Overexpression of vascular endothelial growth factor (VEGF) receptors on keratinocytes in psoriasis: regulated by calcium independent of VEGF. J Cell Mol Med. 2008; 12(2): 649-660, doi: 10.1III/j.15824934.2007.00। I2.x, indexed in Pubmed: 18419602.

31. Jiang M, Li B, Zhang J, et al. Vascular endothelial growth factor driving aberrant keratin expression pattern contributes to the pathogenesis of psoriasis. Exp Cell Res. 2017; 360(2): 310-319, doi: 10.1016/j.yexcr.2017.09.02I, indexed in Pubmed: 28928080.

32. Nielsen HJ, Christensen IJ, Svendsen MN, et al. Elevated plasma levels of vascular endothelial growth factor and plasminogen activator inhibitor-I decrease during improvement of psoriasis. Inflamm Res. 2002; 5 I(II): 563-567, doi: https://doi.org//0.1007/ PL000 I2428, indexed in Pubmed: I254002I.

33. Xuan Ml, Lu Cj, Han L, et al. Circulating levels of inflammatory cytokines in patients with psoriasis vulgaris of different Chinese medicine syndromes. Chinese Journal of Integrative Medicine. 20।4; 2I(2): I08-II4, doi: 10.1007/s I |655-0|4-1792-0.

34. Meki ARMA, Al-Shobaili H. Serum vascular endothelial growth factor, transforming growth factor $\beta I$, and nitric oxide levels in patients with psoriasis vulgaris: their correlation to disease severity. J Clin Lab Anal. 2014; 28(6): 496-50I, doi: 10.1002/ jcla.2 1717, indexed in Pubmed: 24659464.

35. Andrys C, Borska L, Pohl D, et al. Angiogenic activity in patients with psoriasis is significantly decreased by Goeckerman's therapy. Arch Dermatol Res. 2007; 298(I0): 479-483, doi: 10.1007/ s00403-006-0723-8, indexed in Pubmed: 17221216.

36. Akman A, Dicle O, Yilmaz F, et al. Discrepant levels of vascular endothelial growth factor in psoriasis patients treated with PUVA, Re-PUVA and narrow-band UVB. Photodermatol Photoimmunol Photomed. 2008; 24(3): 123-127, doi: 10.1 I I I/j. 16000781.2008.00349.x, indexed in Pubmed: 18477। 30.

37. Weidemann AK, Crawshaw AA, Byrne E, et al. Vascular endothelial growth factor inhibitors: investigational therapies for the treatment of psoriasis. Clin Cosmet Investig Dermatol. 2013; 6: 233-244, doi: 10.2147/CCID.S353/2, indexed in Pubmed: 24101875.

38. Barile S, Medda E, Nisticò L, et al. Vascular endothelial growth factor gene polymorphisms increase the risk to develop psoriasis. Exp Dermatol. 2006; 15(5): 368-376, doi: 10.1 I I //j.09066705.2006.00416.x, indexed in Pubmed: 16630077.
39. Yamamoto M, Tada Y, Asahina A, et al. Severe generalized pustular psoriasis accompanied by bullae formation with increased serum vascular endothelial growth factor level. J Dermatol. 2012; 39(2): 183-185, doi: 10.1III/j.1346-8138.201 I.01235.x, indexed in Pubmed: 21767290.

40. Creamer D, Allen M, Jaggar R, et al. Mediation of systemic vascular hyperpermeability in severe psoriasis by circulating vascular endothelial growth factor. Arch Dermatol. 2002; 138(6): 79|-796, indexed in Pubmed: | 205696I.

4I. Przepiera-Będzak H, Fischer K, Brzosko M. Serum levels of angiogenic cytokines in psoriatic arthritis and $\mathrm{SAPHO}$ syndrome. Pol Arch Med Wewn. 2013; 123(6): 297-302, indexed in Pubmed: 237II57I.

42. Sudhesan A, Rajappa M, Chandrashekar L, et al. Vascular endothelial growth factor (VEGF) gene polymorphisms (rs699947, rs83306I, and rs2010963) and psoriatic risk in South Indian Tamils. Hum Immunol. 2017; 78(10): 657-663, doi: 10.1016/j. humimm.2017.08.004, indexed in Pubmed: 28803785.

43. Young HS, Summers AM, Read IR, et al. Interaction between genetic control of vascular endothelial growth factor production and retinoid responsiveness in psoriasis. J Invest Dermatol. 2006; 126(2): 453-459, doi: 10.1038/sj.jid.5700096, indexed in Pubmed: 16385345.

44. Young HS, Summers AM, Bhushan M, et al. Single-nucleotide polymorphisms of vascular endothelial growth factor in psoriasis of early onset. J Invest Dermatol. 2004; 122(I): 209-215, doi: 10.1046/j.0022-202X.2003.22107.x, indexed in Pubmed: I4962।I0.

45. Hawkes JE, Nguyen GH, Fujita M, et al. microRNAs in Psoriasis. J Invest Dermatol. 2016; 136(2): 365-37I, doi: 10.1038/ JID.2015.409, indexed in Pubmed: 26802234.

46. Li Y, Su J, Li F, et al. MiR-I50 regulates human keratinocyte proliferation in hypoxic conditions through targeting HIF-I and VEGFA: Implications for psoriasis treatment. PLoS One. 2017; 12(4): e0175459, doi: 10.1371/journal.pone.0175459, indexed in Pubmed: 28399173.

47. Zheng YZ, Chen CF, Jia LY, et al. Correlation between microRNA-143 in peripheral blood mononuclear cells and disease severity in patients with psoriasis vulgaris. Oncotarget. 2017; 8(31): 51288-51295, doi: 10.18632/oncotarget. 17260, indexed in Pubmed: 28881648.

48. Xu Y, Ji Y, Lan X, et al. miR203 contributes to ILI 7induced VEGF secretion by targeting SOCS3 in keratinocytes. Mol Med Rep. 2017; 16(6): 8989-8996, doi: 10.3892/mmr.2017.7759, indexed in Pubmed: 29039484.

49. Canavese M, Altruda F, Ruzicka T, et al. Vascular endothelial growth factor (VEGF) in the pathogenesis of psoriasis--a possible target for novel therapies? J Dermatol Sci. 2010; 58(3): 17I-176, doi: 10.1016/j.jdermsci.2010.03.023, indexed in Pubmed: 20430590.

50. Akman A, Yilmaz E, Mutlu $H$, et al. Complete remission of psoriasis following bevacizumab therapy for colon cancer. Clin Exp Dermatol. 2009; 34(5): e202-e204, doi: 10.1 I I //j.13652230.2008.02991.x, indexed in Pubmed: 19077094.

5I. Datta-Mitra A, Riar NK, Raychaudhuri SP. Remission of psoriasis and psoriatic arthritis during bevacizumab therapy for renal cell cancer. Indian J Dermatol. 2014; 59(6): 632, doi: 10.4103/00195I54.143574, indexed in Pubmed: 254844I8.

52. Narayanan S, Callis-Duffin K, Batten J, et al. Improvement of psoriasis during sunitinib therapy for renal cell carcinoma. 
Am J Med Sci. 2010; 339(6): 580-58I, doi: 10.1097/MAJ.0b0I3e3l8Iddlaa5, indexed in Pubmed: 20421784.

53. Fournier C, Tisman G. Sorafenib-associated remission of psoriasis in hypernephroma: case report. Dermatol Online J. 2010; 16(2): 17, indexed in Pubmed: 201787/3.
54. Kuang YH, Lu Y, Liu YK, et al. Topical Sunitinib ointment alleviates Psoriasis-like inflammation by inhibiting the proliferation and apoptosis of keratinocytes. Eur J Pharmacol. 2018; 824: 57-63, doi: 10.1016/j.ejphar.2018.01.048, indexed in Pubmed: 29382533 